\title{
Lenticulostriate Artery Aneurysm Associated with Cerebral Arteriovenous Malformation: Report of Rare Association and Its Endovascular Management
}

\author{
Santhosh Kumar Kannath ${ }^{1}$ Jayadevan Enakshy Rajan ${ }^{1}$ \\ ${ }^{1}$ Department of Imaging Sciences and Interventional Radiology, \\ Neurointervention Center, Sree Chitra Tirunal Institute of Medical \\ Sciences and Technology, Trivandrum, Kerala, India \\ Address for correspondence Jayadevan Enakshy Rajan, DM, \\ Department of Imaging Sciences and Interventional Radiology, \\ Neurointervention Center, Sree Chitra Tirunal Institute of Medical \\ Sciences and Technology, Trivandrum, Kerala 695011, India \\ J Clin Interv Radiol ISVIR 2017;1:114-116. \\ (e-mail: drjayadevan@gmail.com).
}

\author{
Abstract \\ Keywords \\ - lenticulostriate artery \\ aneurysm \\ - cerebral \\ arteriovenous \\ malformation \\ - endovascular \\ embolization
}

Aneurysms involving the lenticulostriate arteries are rare and are described in the literature in small case series or single-case reports. In this report, we discuss a case of a young woman who presented with acute lateral ventricular bleed and was found to have an arteriovenous malformation within the corona radiata and an aneurysm involving the distal lenticulostriate artery. The aneurysm was treated successfully by endovascular embolization. In deep-seated arteriovenous malformations (AVMs) that are conventionally treated by stereotactic radiosurgery, a targeted approach for treatment of an associated aneurysm could be considered to reduce the risk of rebleed until the AVM is obliterated.

\section{Introduction}

Aneurysms involving the lenticulostriate artery (LSA) are rare and are described in the literature in small case series or single-case reports. A recent literature review identified fewer than 60 cases reported till date, and the management of these aneurysms varied from conservative approach to endovascular occlusion and surgical excision. ${ }^{1}$ The literature on endovascular treatment is rare; however, recent studies increasingly report on the usefulness of minimally invasive therapies in the management of these aneurysms.

\section{Case Description}

An 11-year-old female patient with no known comorbidities presented with symptoms of sudden onset severe headache and multiple episodes of vomiting. Computed tomography (CT) scan of the brain revealed an acute small hematoma in the roof of the right lateral ventricle and mild intraventricular bleed. CT angiography was obtained 4 days later, which showed a parenchymal arteriovenous malformation (AVM) involving the corona radiata and the posterior lentiform nucleus. She was initially treated conservatively and was referred to our institution for further management. She underwent digital subtraction angiography after 2 months of clinical presentation. Angiography confirmed the presence of the AVM and identified the feeders from lateral lenticulostriate arteries of right middle cerebral artery (MCA) as well as multiple medullary arteries from the M2 and M3 segments of the MCA. In addition, there was a $5-\mathrm{mm}$ aneurysm at the distal segment of the lateral LSA (LLSA) feeding the AVM. Considering the risk of rupture of the aneurysm and its associated neurologic sequelae, a targeted therapy of the aneurysm with endovascular occlusion was planned prior to stereotactic radiosurgery of the AVM. Under general anesthesia, femoral arterial access was obtained with a 7F arterial sheath, and intravenous heparin was administered to maintain activating clotting time between 200 and 250 seconds. A 6F Envoy guide catheter (Codman \& Shurtleff, Raynham, MA, Massachusetts, United States) was placed in the right petrous internal carotid artery. The LLSA was catheterized using a combination of Marathon microcatheter and Mirage guidewire (Medtronic, Irvine, California, United States). During catheterization, the microcatheter often prolapsed into the main stem of the MCA due to the recurrent origin of the LLSA. The guidewire was advanced sufficiently 
into distal LLSA for stability. The wire slack was removed and was gently torqued to either sides to identify the position that resulted in minimal wire prolapse. While maintaining a counter torque, the microcatheter was then advanced and the LLSA beyond the cisternal segment was catheterized. The microcatheter was positioned immediately proximal to the aneurysm, and the artery along the aneurysm and a part of the nidus was obliterated using 33\% n-butyl cyanoacrylate (nBCA; Histoacryl, B Braun, Melsungen, Germany) (-Fig. 1). Follow-up CT scan of the brain revealed no infarction and she was discharged neurologically intact on the second postoperative day. Follow-up angiogram after 2 months of the embolization revealed no intranidal or feeding artery aneurysms. The residual AVM was treated by stereotactic radiosurgery. The patient remained clinically asymptomatic at 12 months follow-up, and presently, she is considered for repeat angiography to assess response to radiotherapy.

\section{Discussion}

Aneurysms of the LSA are often located within the basal ganglia and hence these aneurysms carry worse prognosis in the event of rupture. Indeed, most of the reported aneurysms presented with parenchymal hemorrhage and the extent of recovery varied. ${ }^{2}$ The aneurysms involving the distal LSA may be associated with moyamoya disease, dissection, or hypertension. However, the majority of these aneurysms are idiopathic. The natural history of these aneurysms is unknown and some aneurysms are known to spontaneously disappear on follow-up studies. This may be true in dissecting aneurysms where the dissected arterial wall heals over a period of time, allowing spontaneous thrombosis, and hence a wait-and-watch policy has been adopted by some authors as one of the therapeutic strategies. ${ }^{2}$ Aneurysms of LSA in association with cerebral AVMs are rare, with only four reported cases so far. Aneurysms in association with cerebral AVMs are found in 3 to $58 \%$ of cases, and the presence of these extranidal aneurysms are thought to pose additional risk of future hemorrhage. ${ }^{3,4}$ Exaggerated hemodynamic stress on the feeding artery and additional underlying intrinsic vascular defects may play a crucial role in the formation of these aneurysms. ${ }^{5}$ Though conservative approach has been advocated for LSA aneurysms per se as well as for the distal feeding aneurysms coexisting with AVM, we considered definitive therapy as the aneurysm had sizeable dimension and the aneurysm was located within the basal ganglia. ${ }^{6}$ Also, a recent study reported an association between the size of the aneurysm and the risk of AVM rupture. ${ }^{4}$

Surgical approaches include exclusion of the aneurysm or parent artery sacrifice; however, these approaches are

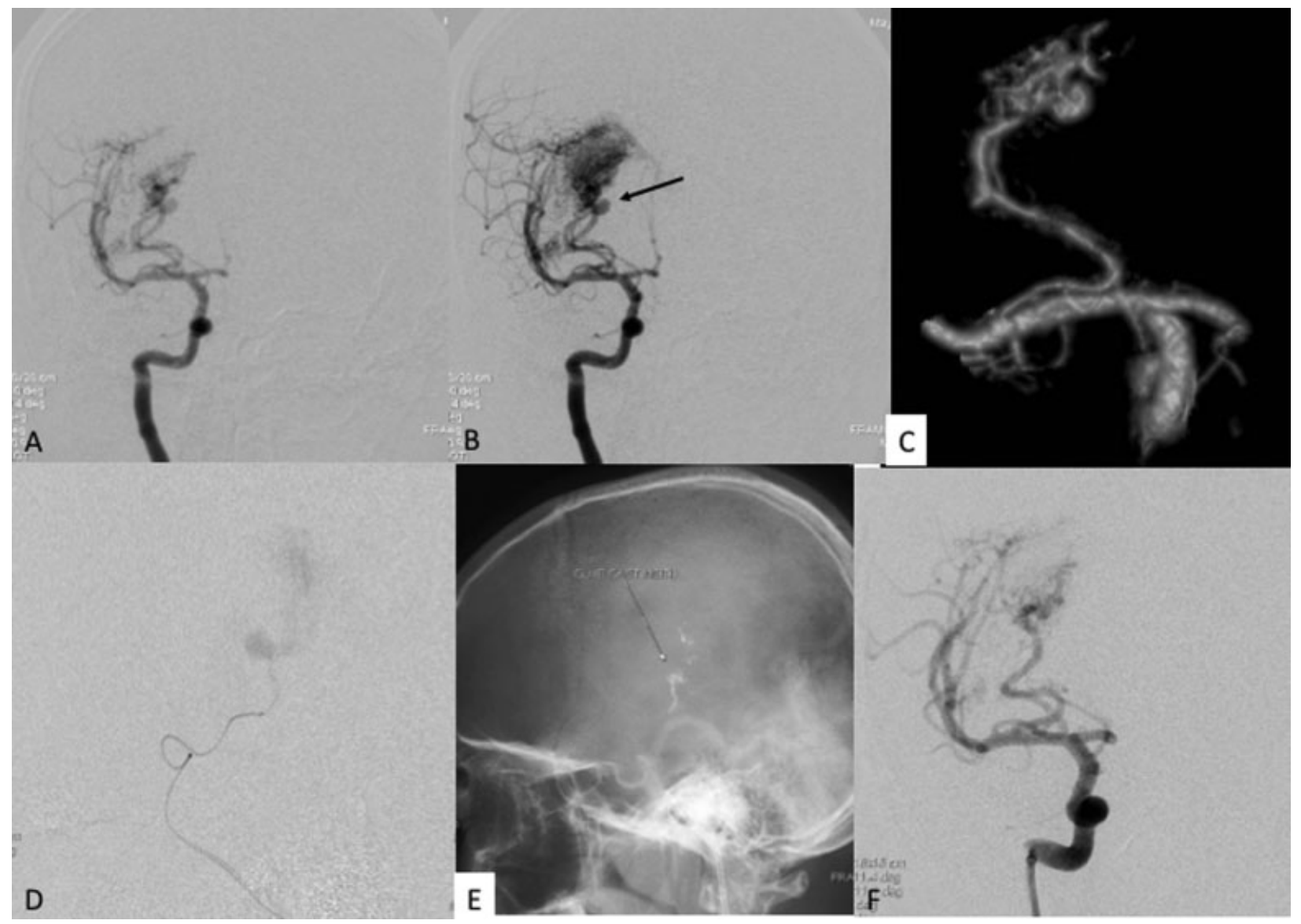

Fig. 1 Diagnostic angiography reveals an arteriovenous malformation within the basal ganglia $(A, B)$ and an aneurysm at the distal aspect of the lenticulostriate artery (LSA) (arrow in B). Three-dimensional rotational angiography depicts the tortuous recurrent course of the LSA (C) that was catheterized. Angiography through the microcatheter confirms the presence of the aneurysm (D) that was treated with occlusion of the LSA with n-butyl cyanoacrylate (E). Final angiography reveals exclusion of the aneurysm (F) and residual arteriovenous malformation. 
fraught with injury to the perforating arteries and infarction of the basal ganglia. Endovascular therapy is also complicated by technical difficulties such as acute origin and small caliber of the LSA, and arterial tortuosity. ${ }^{1,7}$ In our case, the arterial caliber allowed cannulation of the LSA; however, the tortuosity and recurrent origin of the LSA from the MCA resulted in frequent prolapse of the catheter into the MCA. The catheterization was finally performed by a combination of balanced counter torque of the microguidewire and advancement of microcatheter. Occlusion of proximal LSA increases the risk of perforator infarction and hence catheterization of distal segment should be attempted to improve the safety profile of embolization. The endovascular occlusion using embolic materials such as nBCA and onyx is described in the literature. Of the LSA aneurysms associated with AVMs, two cases were treated with surgical excision and two were treated with onyx and nBCA. ${ }^{1}$

Though deep-seated AVMs are associated with higher risk of hemorrhage and require definitive treatment in many instances, achieving a complete cure with acceptable morbidity and mortality remains a therapeutic challenge. Stereotactic radiosurgery is a safe and an effective alternative; however, due to its variable latency period that may extend up to many years, patients could still be susceptible to hemorrhagic risks, especially in the presence of weakened angioarchitectural regions. ${ }^{8}$ Targeted embolization obliterates the focal weak areas, such as the pseudoaneurysms or fistulas within the AVM, and consequently eliminates or reduces the risk of rebleed in the interim period. ${ }^{9}$

Our report suggests that endovascular parent artery occlusion is a safe option in the treatment of LSA aneurysms associated with an AVM. The safety of this procedure can be further improved if intraoperative neurophysiological monitoring is obtained.

\section{Contributions of Authors}

Santhosh Kumar Kannath contributed in concept, design, data analysis, data interpretation, manuscript preparation, and critical revision, and Jayadevan Enakshy Rajan contributed in data analysis, data interpretation, manuscript preparation, and critical revision.

\section{Conflicts of Interest}

The authors have no personal or financial conflict of interest to disclose.

\section{References}

1 Vargas J, Walsh K, Turner R, Chaudry I, Turk A, Spiotta A. Lenticulostriate aneurysms: a case series and review of the literature. J Neurointerv Surg 2015;7(03):194-201

2 Lama S, Dolati P, Sutherland GR. Controversy in the management of lenticulostriate artery dissecting aneurysm: a case report and review of the literature. World Neurosurg 2014;81(02):441.e1441.e7

3 Kim EJ, Halim AX, Dowd CF, et al. The relationship of coexisting extranidal aneurysms to intracranial hemorrhage in patients harboring brain arteriovenous malformations. Neurosurgery 2004;54(06):1349-1357, discussion 1357-1358

4 Stein K-P, Wanke I, Forsting M, et al. Associated aneurysms in supratentorial arteriovenous malformations: impact of aneurysm size on haemorrhage. Cerebrovasc Dis 2015;39(02): 122-129

5 Stapf C, Mohr JP, Pile-Spellman J, et al. Concurrent arterial aneurysms in brain arteriovenous malformations with haemorrhagic presentation. J Neurol Neurosurg Psychiatry 2002;73(03): 294-298

6 Redekop G, TerBrugge K, Montanera W, Willinsky R. Arterial aneurysms associated with cerebral arteriovenous malformations: classification, incidence, and risk of hemorrhage. J Neurosurg 1998;89(04):539-546

7 Chalouhi N, Tjoumakaris S, Gonzalez LF, et al. Onyx embolization of a ruptured lenticulostriate artery aneurysm in a patient with moyamoya disease. World Neurosurg 2013;80(3-4):436.e7436.e10

8 Kano H, Kondziolka D, Flickinger JC, et al. Stereotactic radiosurgery for arteriovenous malformations, Part 4: management of basal ganglia and thalamus arteriovenous malformations. J Neurosurg 2012;116(01):33-43

9 Krings T, Hans F-J, Geibprasert S, Terbrugge K. Partial “targeted" embolisation of brain arteriovenous malformations. Eur Radiol 2010;20(11):2723-2731 